\title{
Palliative sedation to alleviate existential suffering at end-of-life: Insight into a controversial practice
}

\section{by Kim Sadler}

\begin{abstract}
All terminally ill individuals should have access to palliative care that gives equal importance to all aspects of suffering. In spite of all the efforts made, some of these individuals will, unfortunately, be left with suffering described as "refractory". It would appear that palliative sedation then becomes an option. There is much controversy around this practice, especially when it is used to alleviate suffering of a more existential nature. This article aims first to define the practice of palliative sedation, second to explore the concept of existential suffering in palliative care and third to address the implications for nursing practice.
\end{abstract}

Ms. B., 29, is living with endometrial cancer. Despite surgery and the numerous chemotherapy treatments she has received since diagnosis, medical imaging tests reveal the illness' continuing progress. Multiple metastases have spread to the intestinal walls. Presenting with a complete bowel obstruction, she has been admitted to the palliative care unit. A nasogastric tube attached to suction is in place to prevent vomiting and has confined her to bed. The severe pains and nausea that were initially present are now under control. Her devastated parents remain at her bedside 24/7. Ms. B. has lost more than 30 pounds in the last month and presents with generalized weakness. She says she feels like she has become a burden for her family, she is living a pointless life and, above all, she is no longer herself. She confides that seeing herself dying has become intolerable to her. A psychiatrist and a psychologist have been consulted. Because the clinical presentation does not match anything mentioned in the Diagnostic and Statistical Manual for Mental Disorders (DSM-IV-TR), no diagnosis of depression or any other mental disorder has been established. Her prognosis is estimated to be a few weeks. At Ms. B.'s request, a continuous infusion of a sedative has been initiated. This infusion will keep her in a lethargic state until she dies.

Sophie, a nurse, has been finding it difficult to care for Ms. B. since palliative sedation was induced. She dares not talk about it, but feels like she is participating in some form of assisted suicide and wonders if there could not have been some other less extreme options...

All terminally ill individuals should have access to palliative care that gives equal importance to the physiological, as well as psychosocial and spiritual aspects of suffering (Quill \& Byock, 2000). In spite of all the efforts made, some individuals are left

\section{About the author}

Kim Sadler, BScN, PhD(c) Research/Intervention in Clinical Psychology. Email: kim.sadler@umontreal.ca struggling with unresolved symptoms at end of life. These symptoms are called "refractory" in the palliative care context. These can be pain, nausea, vomiting, delirium and bleeding, as well as psychological or existential distress. The main characteristic of a refractory symptom is that it has become intolerable. In the palliative care context, it seems that when all available interventions to alleviate a symptom have failed, one last option emerges, that of palliative sedation. So palliative sedation is seen as a type of last resort therapy (De Graeff \& Dean, 2007; Muller-Bush, Andres, \& Jehser, 2003; Quill \& Byock, 2000; Schuman-Olivier, Brendel, Forstein, \& Price, 2008). Several proposed guidelines have been published in order to make this practice more structured, ethical, justified and, above all, to differentiate it from assisted suicide and euthanasia. Nevertheless, its use still gives rise to much controversy. This is mostly due to a lack of clear and universally accepted definitions of what palliative sedation (Maltoni et al., 2009; Morita, Tsuneto, \& Shima, 2002) and refractory symptoms really are (Morita, Tsuneto, \& Shima, 2002). The boundaries with the practice of assisted suicide and euthanasia are not clearly defined for many, especially when the refractory symptom at play relates to existential suffering.

This article aims first to define the practice of palliative sedation, second to explore the concept of existential suffering in palliative care and third to address the implications for nursing practice.

\section{Palliative sedation}

In the context of this paper, palliative sedation is defined as the use of sedative medications to relieve unendurable suffering from refractory symptoms by a reduction in the level of consciousness (De Graeff \& Dean, 2007). It is difficult to estimate the prevalence of this practice given the inconsistencies in its definition and disparities in its enumeration (De Graeff \& Dean, 2007). Palliative sedation goes by a number of different names in the literature, which contributes to maintaining some confusion around the nature of its practice. The following are but a few examples: total sedation, sedation for refractory symptoms, continuous deep sedation, palliative sedation therapy and terminal sedation. Some categorize it according to its schedule (e.g., intermittent, continuous), the level of sedation (e.g., light, moderate, deep), the type of medication used (e.g., benzodiazepines, antipsychotics). The practice that remains the most controversial is the use of continuous deep sedation, which is maintained until the time of death (Rietjens, Buiting, Pasman, van der Maas, van Delden, \& van der Heide, 2009).

Regarding the "intolerable" nature of a symptom, Cherny (1994) put forward an interesting description. It is one that cannot be adequately controlled despite aggressive efforts to identify a tolerable therapy that does not compromise consciousness; moreover, additional interventions would not provide adequate relief within an acceptable timeframe in these cases. Despite the operationalization 
of the concept, there remains a significant degree of subjectivity. Only the patients themselves can decide on the intractable character of a symptom (Morita, Tsuneto, \& Shima, 2002). After all, they are the ones experiencing their suffering.

Some argue that this type of sedation is a disguised form of assisted suicide or euthanasia, especially when the refractory symptom in play relates to existential suffering (Rady \& Verheijde, 2010). Many articles have been written in the last few years to demonstrate that these practices are separate and particularly that palliative sedation does not constitute a slippery slope towards euthanasia. Intent, method used and goal are the most often quoted criteria to differentiate it from assisted suicide and euthanasia (Muller-Bush, Andres, \& Jehser, 2003). In the context of palliative sedation, the intent is to relieve suffering, most often through sedative drugs (e.g., benzodiazepines) and the goal is to provide relief. In the case of euthanasia, the intent is to cause rapid death through the use of lethal medication (Muller-Bush, Andres, \& Jehser, 2003). Some researchers claimed that palliative sedation did not hasten the dying process in terms of survival time for the terminally ill (e.g., the number of days) and drew on these results and others to differentiate between this practice and that of euthanasia (Maltoni et al., 2009; National Ethics Committee, 2007; Muller-Bush, Andres, \& Jehser, 2003).

The decision to start continuous deep sedation, especially when it is to overcome existential suffering, when the individual is often conscious and their physiological symptoms are relatively well relieved, is a difficult one. The cultural, religious and social values of clinicians are being questioned, and the decision process is fraught with strong emotions. The decision to adopt such a measure presents the clinicians with a significant dilemma. According to their professional dictates, their duty is to alleviate suffering, not precipitate death. These are two axioms of their profession. Faced with such a conflict when considering palliative sedation, clinicians will often choose to turn to the law and ethical rules for guidance.

\section{The law}

The debate continues in spite of the fact the Supreme Court of the United States approved the practice of sedation in 1997 (Schuman-Olivier, Brendel, Forstein, \& Price, 2008; Crenshaw, 2009). In two judgements regarding assisted suicide (Vacco v. Quill) (Washington v. Glucksberg), the Supreme Court seemed to distinguish palliative sedation from assisted suicide and, furthermore, to deem it the only legally acceptable practice (National Ethics Committee, 2007). The Court did not explicitly mention palliative sedation, but stated nonetheless its support of the use of medication to alleviate pain and suffering in terminally ill individuals even if it could render them unconscious and possibly hasten the dying process (National Ethics Committee, 2007). In the Canadian arena, in the Rodriguez v. British Columbia (Attorney General) decision, the Court of Appeal states that several medical organizations seem to approve the use of medication to relieve pain in palliative care while acknowledging that this may sometimes hasten death. These medical organizations specify that the primary intent is still to alleviate pain and mental suffering in order to allow death without excessive suffering rather than hasten it. One needs to know that Ms. Rodriguez appealed this decision to the Supreme Court of Canada in $R$. v. Rodriguez. Without adjudicating on the issue of palliative sedation, the Supreme Court considered that in Western democracies, including Canada, assisted suicide is prohibited. The reason is these states "recognize and apply the principle of the sanctity of life as a general principle which is subject to limited and narrow exceptions in situations in which notions of personal autonomy and dignity must prevail." The concepts of autonomy and dignity are consistently invoked in decisions as to whether or not to induce palliative sedation for unendurable suffering. This practice is still being debated, partly because of differences of opinion as to what these legal and philosophical concepts stand for. For example, does dying while afflicted with physical or psychic suffering make an individual unworthy? May individuals reaching the end of their lives freely decide to spend the rest of their days in an iatrogenic coma?

\section{Ethics}

Several ethical principles are frequently cited to justify the use of palliative sedation, mainly the following: the doctrine of double effect, the principle of proportionality, and the principle of autonomy (Morita, 2003).

\section{Doctrine of double effect}

According to this doctrine, a single act may have two distinct outcomes: a good one and a negative one, hence its name. The doctrine's tenets usually require four criteria to be met: (1) The nature of the act must be good or morally neutral, (2) the good effect must result from the act itself and not from the negative outcome, (3) the negative outcome must not be directly sought, but can be foreseen and tolerated, (4) there must be proportionality between the good and the negative outcomes; the good one must exceed the negative one or at least balance it (Crenshaw, 2009; Schuman-Olivier, Brendel, Forstein, \& Price, 2008). Lo and Rubenfeld (2005) put forward a fifth criteria in the context of the decision making regarding initiation of sedation: (5) There is no other option that would be less harmful. There is little consensus on invoking the double effect principle to justify the use of palliative sedation. For example, it was suggested that it is rather difficult to make inferences regarding the intents of a third party (Lo \& Rubenfeld, 2005). In a study conducted in the Netherlands, $47 \%$ of a group of more than 200 physicians recognized having intended-in part-to hasten the dying process, and 17\% having had explicitly wanted to cause death by resorting to terminal sedation (Rietjens, van der Heide, Vrakking, Onwuteaka-Philipsen, van der Maas \& van der Wal, 2004). Moreover, still as a criticism to using the principles of double effect, some do not make any distinction between an action resulting in both a good effect and a negative effect if the negative effect was foreseeable and, generally, society tends to condemn individuals for their actions if their effects were forseeable (Lo \& Rubenfeld, 2005).

\section{Principle of proportionality}

In the current context, proportionality refers to a situation where the suffering experienced by an individual is so intractable that resorting to palliative sedation is a justified medical act. In certain situations where suffering is particularly unendurable, one does not turn a blind eye to the risks associated with sedation as a possibility to hasten the dying process, but one expects the benefits to outweigh the risks. In order to assess proportionality of a practice in palliative care, the following criteria can be used: presence of a terminal illness, urgent need to relieve suffering and of course, informed consent freely given by the individual or their representative (McIntyre, 2004). A criticism addressed to the use of this ethical principle in the specific context of sedation in palliative care is the fact that the prognostic assessment is usually not very precise, which means it is difficult to state categorically whether or not death is really "imminent" (Rady \& Verheijde, 2010). This prognosis is even harder to formulate when it concerns end-of-life patients with pulmonary or cardiac affections (National Ethics Committee, 2007). Is resorting to sedation as ethical and justifiable if it turns out the individual's prognosis is longer than predicted? 


\section{Principle of autonomy}

The principle of autonomy refers to the capacity of an individual to make free and informed choices and to act accordingly. Regarding the global issues of palliative sedation, it is up to the individual to give his/her consent while having the necessary information about the practice's benefits and risks, if he/she is deemed to be capable and competent. Only in extraordinary cases can a patient representative make this decision. In various judgments, the Canadian justice system determined that based on the principle of autonomy, an individual is free to refuse any treatment, even if this decision can result in hastening the dying process. For example, if an individual chooses to stop eating and if he/she is then forced to take foods and fluids, this could be considered a form of assault (Quill \& Byock, 2000). Nevertheless, despite the importance given to respecting an individual's choices, the principle of autonomy does not require anyone to engage in practices that are in breach of laws or go against their own moral principles (Rousseau, 2001).

The practice of inducing unconsciousness in the terminally ill in the presence of a refractory symptom of a "physiologic" nature (i.e. pain, delirium, myoclonus) appears to be more readily accepted in care environments than when the refractory symptom is of a "psychological" or "existential" nature (Bruce \& Boston, 2011; Lo \& Rubenfeld, 2005). In the first scenario, the clinician seems to have easier access to visible and objective signs of an individual's suffering while in the second it is much harder to objectively establish the refractory nature of the suffering (Morita, Tsudona, Inoue, \& Chihara, 2000). For physiological symptoms, there seem to exist more guidelines regarding interventions to be attempted before declaring the symptom to be refractory. In contrast, there are currently no clear guidelines on how to characterize existential suffering as refractory in the specific context of palliative care.

Schuman-Olivier, Brendel, Forstein and Price (2008) pointed out that at the time of their study not one article in the field of psychiatry had focused on the current debate about resorting to palliative sedation to alleviate existential distress. When dealing with this type of suffering, the care team and the family face the daunting challenge of distinguishing what could be qualified as normal existential concerns at end of life and what appears to be a more severe, perhaps even a "pathological" form of existential distress requiring more aggressive interventions. Controversy around deep sedation in this context is always present, as this practice refers to death in some way. It may not hasten the dying process to the extent survival time may not be affected by inducing a varying degree of continuous deep sedation, but, by inducing unconsciousness in individuals, aren't we cutting them off from themselves and their world? Isn't this a form of social death?

Furthermore, when sedation is utilized to alleviate existential suffering, at what point does this practice start to reveal how helpless we feel, as caregivers, before an unknown, which appears to be totally out of our control? The alleviation of existential suffering may be partly hindered by the nurse's own vulnerabilities, as she is confronted with her patient's pain. It may be that the fear of the unknown, death and suffering cause some form of distress in clinicians, including nurses, which motivates them to act rapidly to bring relief to the individual, of course, but also to a part of themselves.

\section{Existential suffering}

Some psychological disorders are more prevalent in the terminally ill than in the general population (Block, 2000; APES, 2008). In a few papers, an attempt was made to differentiate between existential suffering per se and another type described as "psychological" suffering (e.g., depression, anxiety) (Block,
2000; Schuman-Olivier, Brendel, Forstein, \& Price, 2008). While this article does not aim to exhaustively clarify how specific psychological disorders present at end of life, it is important to point out that the boundaries between psychological distress (e.g., refractory depression) and existential distress are poorly defined. There is most likely some overlapping between the two, as existential suffering does not constitute a distinct entity, but is more akin to a complex mixture. A thorough assessment of distress is to be conducted by the nurse, as interventions may vary according to the type of suffering. Certain elements can sometimes guide the nurse, as she must be able to distinguish between a psychological disorder and suffering of a more existential nature.

\section{Depression}

To a certain extent, it is not surprising for individuals facing the end of their lives to experience some mood disturbances. After all, these individuals must deal with a number of losses while facing uncertainties relating to the dying process. When one wants to establish a diagnosis of depression in a terminally ill individual, the difficulty lies in that most somatic symptoms associated with depression, as described in the Diagnostic and Statistical Manual for Mental Disorders (DSM-IV-TR), result frequently from the progression of the terminal illness (e.g., loss of appetite, fatigue, weight loss). In this context, it is suggested to rely more on the strictly "psychological" symptoms of depression to establish a diagnosis such as persistent depressive mood, loss of interest and pleasure, guilt feeling, lower self-esteem and continuous suicidal ideation (APES, 2008). In the presence of existential suffering, by contrast with clinical depression, it is possible that the individual has not completely lost interest for all activities and that guilt feeling may be absent, too. Despite these markers, differentiating between depression and existential distress may be a difficult task (Schuman-Olivier, Brendel, Forstein, \& Price, 2008).

\section{Anxiety}

A certain increase in the anxiety level is not unusual but, contrary to some beliefs, an elevated anxiety level is not unavoidable in the terminal phase and it should not be tolerated. In palliative care, it is important to assess the potential presence of anxiety disorders (e.g., acute stress disorder, anxiety disorder due to a general medical condition), particularly because of the higher prevalence of these disorders in this population, but also because when it is left untreated, the anxiety disorder may bring the subject to ask that the dying process be hastened. Beyond its association with an anxiety disorder, it is critical to be mindful that anxiety may be the evidence of poorly managed physiological symptoms (e.g., pain, dyspnea, urinary retention) or even of medication or treatment side effects. Existential suffering may generate significant anxiety, just as psychological suffering does.

The difficulties encountered in resorting to sedation to relieve existential suffering come in a large part from the lack of conceptual models for the nature of this suffering. There do exist a few guides and "guidelines" regarding the utilization of palliative sedation to alleviate refractory existential suffering. However, as Bruce and Boston (2011) point out, these guides assume one already knows about the nature of existential suffering, the ways to identify it and deal with it, but this is not supported in the literature.

There are a limited number of papers on existential suffering in the context of palliative and oncology care (Blinderman \& Cherny, 2005). The work of Dame Cicely Saunders, the founder of the modern hospice movement in England, in the 1960s had a considerable influence on the management of individuals facing 
a terminal illness. She introduced the concept of total pain, which implied that all aspects of an individual (e.g., psychological, social, spiritual) may be affected by an incurable disease and that to provide adequate relief one must relieve the whole being through a holistic approach (Saunders, 1988). Subsequently, several authors attempted to investigate the nature of existential suffering in the end-of-life context. Cassel (1982) wrote that suffering can be defined as a state of severe distress associated with events that threaten the intactness of the person. Existential concerns are frequently present among cancer patients (Blinderman \& Cherny, 2005). They can relate to various issues such as hopelessness, futility, meaninglessness, disappointment, remorse, death anxiety and disruption of personal identity (Blinderman \& Cherny, 2005). Kearny (2000) defined suffering as an experience resulting from an attack on the whole person. After completing a literature review, Morita (2004a) suggested recurring themes for the sources of existential suffering including meaninglessness of current life, loss of social roles, feeling of dependency, fear of being a burden for others, guilt, incomplete projects. Many believe that experiencing existential concerns at end of life is universal, but these concerns do not automatically engender distress in everyone (Chochinov, 2006). Kissane (2001) suggested the demoralization syndrome as a psychiatric diagnosis to identify existential distress. This syndrome consists in: 1) hopelessness, loss of meaning, and existential distress, 2) pessimism, powerlessness, sense of being trapped, personal failure, lack of a worthwhile future, 3) absence of motivation to cope differently, 4) isolation and lack of support, 5) duration of more than two weeks, and 6) lack of a major depression diagnosis or other psychiatric diagnoses. Despite the rich contributions from all these studies, there is still no universally accepted definition in the literature. Without a common language, it is hard to develop interventions and justify the use of such an extreme practice as palliative sedation.

Much of the current research on suffering is grounded in philosophical concepts. Several existentialist thinkers of the 19 th and 20th centuries reflected primarily on the conditions of existence and human suffering. Existentialists emphasize the subjective experience of each individual. Human beings come into existence first, and then create their own essence out of interaction with their surroundings and among themselves. Differences encountered amidst the supporters of this philosophical approach rest mainly upon the way each individual chooses to make sense out of his/her life, the potential obstacles to attaining his/her goals and the involvement, or not, of God along this path. The main themes addressed by existentialists are futility of life, indifference, anguish, boredom, alienation, freedom and nothingness. Concerning death, man ceases to be and the only way he can continue to exist is in the memories of others. He does not suffer either, as anguish does not belong to death but to living. A further exploration of these writings will contribute to a deeper understanding of the sources of existential suffering.

\section{Implications for nursing practice}

Initiating continuous deep sedation may be a difficult experience for nurses (Morita, Miyashita, Kimura, Adachi, \& Shima, 2004b). Nurses play a central role as patient advocates throughout the health care system (Bruce \& Boston, 2011). However, in situations as emotionally charged as the one depicted in the clinical vignette at the beginning of this article, nurses can end up questioning the moral implications of their role as caregivers. Jameton (1984) talks about the notion of moral uncertainty. Taking part in interventions like palliative sedation where nurses are unsure it constitutes the best option is likely to confront nurses with this kind of uncertainty. This can give rise to discomfort, stress and even a state of frustration (Cohen \& Erikson, 2006). There are few nursing science papers about the use of palliative sedation (Bruce \& Boston, 2011). Nurses are invited to take a greater part in the current debate around this practice (Bruce $\&$ Boston, 2011). Given the fact they are the caregivers who generally spend the most time with the patient in comparison with the other care team members, it is paramount that nurses be involved in decision processes (Crenshaw, 2009).

Furthermore, at the bedside of the dying, nurses often find themselves confronting their own vulnerability concerning illness, suffering and death. They can protect themselves by distancing themselves from their patients. They are not always aware that their own sources of distress can become obstacles to address the suffering of others. Nurses must regularly consider the impact that the strong emotions such as fear and helplessness they feel at the sight of a suffering patient may have on the decision to initiate or participate in palliative sedation. The decision is most likely based on a desire to calm a suffering patient, but couldn't there also be more or less conscious motivations to want to calm themselves, as caregivers confronted with pains that defy them? It is also relevant to stress at this time the little importance granted to the the phenomenon of suffering in the general nursing training curriculum. While learning about relieving physical pain remains largely unaddressed, the theme of suffering simply goes unmentioned. It's not a matter of developing guidelines to address existential suffering at end of life, as this would be completely counterproductive. Suffering defies objectivity. Rather, it is a matter of promoting greater awareness among nurses about the importance of people-oriented skills over know-how in the specific context of end-of-life care. It is also a question of helping nurses distinguish their own distress from that of their patients so that they can improve the quality of their presence by their side in the closing moments of their lives. Moreover, the organization must promote an atmosphere where nurses feel heard, supported and respected in their opinions and choices.

To conclude, palliative sedation is an extreme measure that remains controversial, particularly when it is utilized to alleviate existential distress as it refers to the practice of assisted suicide and euthanasia. Through different means, these three practices cut off individuals from themselves, from their environment and from time. Palliative sedation is considered to be a last resort method of relieving suffering. We rely on the laws that permit it and on certain ethical principles that guide it but, to linger on the issue, aren't we turning our attention away from the real problem, that is to say, what is this existential suffering that we are trying to stun into unconsciousness? Few studies have dwelt on the matter, leaving nurses feeling helpless in front of such suffering. The danger regarding the use of palliative sedation is that it could be considered an easy way of providing quality palliative care without having to consider all aspects of suffering, including its existential one. In a study conducted by Blondeau, Roy, Dumont, Godin and Martineau (2005) about the attitudes of physicians and pharmacists toward the use of sedation at the end of life, one participant had asked a question worth thinking about: Can the lack of a meaningful existence be treated with sleep?"

\section{Acknowledgements}

The author would like to thank David K. Wright, Dr. H. Chang and Dr. D. Scarfone for their contributions to the thinking process. 


\section{REFERENCES}

APES (2008). Guide pratique des soins palliatifs: gestion de la

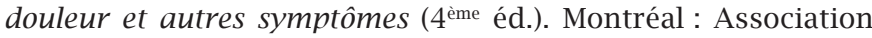
des pharmaciens des établissements de santé du Québec (APES).

Blinderman, C.D., \& Cherny, N.I. (2005). Existential issues do not necessarily result in existential suffering: Lessons from cancer patients in Israel. Palliative medicine, 19, 371-380.

Block, S.D. (2000). Assessing and managing depression in terminally ill patients. Annals of Internal Medicine, 132(3), 209-218.

Blondeau, D., Roy, L., Dumont, S., Godin, G., \& Martineau, I. (2005). Physicians' and pharmacists' attitudes toward the use of sedation at the EOL: Influence of prognosis and type of suffering. Journal of Palliative Care, 21(4), 238-245.

Bruce, A., \& Boston, P. (2011). Relieving existential suffering through palliative sedation: Discussion of an uneasy practice. Journal of Advanced Nursing, 67(12), 2732-2740.

Cassel, E.H. (1982). The nature of suffering and the goals of medicine. New England Journal of Medicine, 306, 639-645.

Cherny, N.I., Coyle, N., \& Foley, K.M. (1994). Suffering in the advanced cancer patient: A definition and taxonomy. Journal of Palliative Care, 10, 57-70.

Chochinov, H.M. (2006). Dying, dignity, and new horizons in palliative end-of-life care. CA Cancer Journal for Clinicians, 56, 84-103.

Cohen, J.S., \& Erickson, J.M. (2006). Ethical dilemmas and moral distress in oncology nursing practice. Clinical Journal of Oncology Nursing, 10(6), 775-779.

Crenshaw, J. (2009). Palliative sedation for existential suffering. Journal of Hospice and Palliative Nursing, 11(2), 101-106.

De Graeff, A., \& Dean, M. (2007). Palliative sedation therapy in the last weeks of life: A literature review and recommendations for standards. Journal of Palliative Medicine, 10(1), 67-85.

Jameton, A. (1984). Nursing practice: The ethical issues. Englewood Cliffs. NJ: Prentice Hall.

Kearny, M. (2000). A place of healing: Working with suffering in living and dying. Oxford: Oxford University Press.

Kissane, D.W., Clarke, D.M., \& Street, A.F. (2001). Demoralization syndrome-A relevant psychiatric diagnosis for palliative care. Journal of Palliative Care, 17, 12-21.

Lo, B., \& Rubenfeld, G. (2005). Palliative sedation in dying patients: "We turn to it when everything else hasn't worked". JAMA, 294(14), 1810-1816.

Maltoni, M., Pittureri, C., Scarpi, E., Piccinini, L., Martini, F., Turci, P., et al. (2009). Palliative sedation therapy does not hasten death: Results from a prospective multicenter study. Annals of Oncology, 20(7), 1163-1169.

McIntyre, A. (2004). The double life of double effect. Theoretical Medicine and Bioethics, 25, 61-74.
Morita, T., Tsudona, J., Inoue, S., \& Chihara, S. (2000). Terminal sedation for existential distress. American Journal of Hospice and Palliative Medicine, 17(3), 189-195.

Morita, T., Tsuneto, S., \& Shima, Y. (2002). Definition of sedation for symptom relief: A systematic literature review and a proposal of operational criteria. Journal of Pain and Symptom Management, 24(4), 447-453.

Morita, T. (2003). Ethical validity of palliative sedation therapy (letter to the editor). Letters, 25(2), 103-104.

Morita, T. (2004a). Palliative sedation to relieve psycho-existential suffering of the terminally ill cancer patients. Journal of pain and symptom management, 28(5), 445-450.

Morita, T., Miyashita, M., Kimura, R., Adachi, I., \& Shima, Y. (2004b). Emotional burden of nurses in palliative sedation therapy. Palliative Medicine, 18, 550-557.

Muller-Bush, H.C., Andres, I., \& Jehser, T. (2003). Sedation in palliative care-A critical analysis of 7 years experience. $B M C$ Palliative Care, 2(2).

National Ethics Committee, Veterans Health Administration. (2007). The ethics of palliative sedation as a therapy of last resort. American J of Hospice and Palliative Medicine, 23(6), 483-491.

Quill, T.E., \& Byock, I.R. (2000). Responding to intractable terminal suffering: The role of terminal sedation and voluntary refusal of food and fluids. Annals of Internal Medicine, 132(5), 408414.

Rady, M.Y., \& Verheijde, J.L. (2010). Continuous deep sedation until death: Palliation or physician-assisted death. American Journal of Hospice and Palliative Care, 27(3), 205-214.

Rietjens, J.A., van der Heide, A., Vrakking, A.M., OnwuteakaPhilipsen, B.D., van der Maas, P.J., \& van der Wal, G. (2004). Physician reports of terminal sedation without hydration or nutrition for patients nearing death in Netherlands. Annals of Internal Medicine, 141(3), 178-185.

Rietjens, J.A., Buiting, H.M., Pasman, H.R.W., van der Maas, P.J., van Delden, J.J.M. \& van der Heide, A. (2009). Deciding about continuous deep sedation: Physicians' perspectives-A focus group study. Palliative Medicine, 23, 410-417.

Rodriguez v. British Columbia (Attorney General), [1993] 3 S.C.R. 519.

Rousseau, P. (2001). Existential suffering and palliative sedation: A brief commentary with a proposal for guidelines. American $J$ of Hospice and Palliative Care, 18(3), 151-153.

Saunders, C. (1988). Spiritual pain. Journal of Palliative Care, 4, 29 32.

Schuman-Olivier, Z., Brendel, D.H., Forstein, M., \& Price, B.H. (2008). The use of palliative sedation for existential distress: A psychiatric perspective. Harvard Review of Psychiatry, 16(6), 339-351. 